Artículo

\title{
Estabilidad del explante en la proliferación de brotes axilares in vitro de la biznaga
}

\author{
Eulalia Edith Villavicencio-Gutiérrez ${ }^{1 \S}$ \\ Gregorio Arellano-Ostoa ${ }^{2}$ \\ Miguel A. Carranza-Pérez ${ }^{3}$
}

${ }^{1}$ Campo Experimental Saltillo-INIFAP. Carretera Saltillo-Zacatecas km 342+119 núm. 9515, Col. Hacienda de Buenavista, Saltillo, Coahuila. CP. 25315. Tel. 800 0882222, ext. 83505. ${ }^{2}$ Programa de Recursos Genéticos, Productividad y Fruticultura-Colegio de Postgraduados. Carretera México-Texcoco km 36.5, Montecillo, Estado de México. CP. 56230. Tel. 595 9520200, ext. 1117. (arellano@colpos.mx). ${ }^{3}$ Departamento de Botánica-Universidad Autónoma Agraria Antonio Narro. Calzada Antonio Narro 1923, Col. Buenavista, Saltillo, Coahuila. CP. 25315. Tel. 844 4110200, ext. 2253. (mcarper.uaaan@ gmail.com).

$\S^{\S}$ Autora para correspondencia: vedithgtz@gmail.com.

\section{Resumen}

Para conservar ex situ, promover el uso y la potenciación de germoplasma nativo de interés ornamental como $T$. viereckii subsp. major una especie en estatus de riesgo Pr, se planteó la presente investigación con el objetivo de regenerar esta especie en el laboratorio de cultivo de tejidos vegetales (LCTV) del Campo Experimental Saltillo CIRNE-INIFAP considerando la estabilidad del explante durante subcultivos subsecuentes, determinar el tipo y la concentración de fitohormonas y la mejor relación citocinina-auxina que más influyen en la etapa de multiplicación in vitro. El segmento intermedio del tallo (SIT) obtenido a partir de vitroplantas se usó como explante. De 2017 a 2019 se evaluó la etapa de multiplicación utilizando el medio MurashigeSkoog al 50\% de sus macrosales. Un diseño experimental completamente al azar con arreglo factorial, se usó para evaluar dos citocininas: 6-bencil aminopurina (BAP) y 6-furfuryl aminopurina (KIN) en cuatro concentraciones 2.5, 5, 7.5 y $10 \mathrm{mg} \mathrm{L}^{-1}$ en interacción con dos auxinas: ácido naftalenacético (ANA) y ácido indolbutírico (AIB) en una relación citocinina-auxina de 10:1. Se evaluaron 16 tratamientos estableciendo cinco SIT/frasco con 10 repeticiones/tratamiento. Esta evaluación se repitió cinco veces mediante subcultivos, evaluando cada diez semanas el número de brotes/explante $(\mathrm{Nb})$ y la altura del brote $(\mathrm{Ab}, \mathrm{mm})$. El análisis de varianza (Anova) demostró que hubo estabilidad del explante durante los subcultivos manteniendo una tasa de multiplicación semejante por más de tres años, efecto importante para una multiplicación intensiva. Al analizar los tratamientos como efectos independientes en la prueba de medias de Tukey $(p \leq 0.05)$, se seleccionó la interacción BAP: AIB y KIN: AIB con las concentraciones de $5 \mathrm{mg} \mathrm{L}^{-1}$ de BAP + 0.5 $\mathrm{mg} \mathrm{L}^{-1}$ de AIB y $2.5 \mathrm{mg} \mathrm{L}^{-1}$ de $\mathrm{KIN}+0.25 \mathrm{mg} \mathrm{L}^{-1}$ de AIB como los tratamientos que inducen brotes con una tasa de multiplicación de 9.25 brotes/explante con una $A b$ de $6.73 \mathrm{~mm}$.

Palabras clave: cactáceas de ornato, cultivo in vitro, reguladores de crecimiento.

Recibido: octubre de 2021

Aceptado: enero de 2022 


\section{Introducción}

El avance en el conocimiento de la regulación hormonal in vitro ha permitido desarrollar protocolos de micropropagación en especies y cultivares de importancia económica como en rosa híbrida Mimosa pudica L. (Bianchetti et al., 2017); (Aggarwal et al., 2018), hasta en algas rojas (Villanueva et al., 2013). El efecto de diferentes concentraciones de fitohormonas en la micropropagación de especies de la familia Cactaceae se ha estudiado en germoplasma en estatus de riesgo de interés para la horticultura ornamental como: en varias especies del género Coryphanta spp. (Pérez-Molphe-Bach et al., 1998), el viejito Cephalocereus senilis (Haw.) Pfeiff. (Choreño et al., 2002), biznaga nido de golondrina Epithelantha micromeris (Engelm.) F. A. C. Weber ex Britt. \& Rose (Villavicencio et al., 2012), mamilaria Mammillaria voburnensis Scheer (Ordoñez, 2003), cactus alcachofa Obregonia denegrii Frič \& A. Berger (Cardarelli et al., 2010), diversos minicactus del género Turbinicarpus spp. (Mata-Rosas et al., 2001; Dávila et al., 2005; Villavicencio et al., 2011) y pitaya Stenocereus stellatus (Pfeiff.) Riccob. (Martínez-Villegas et al., 2011), donde los brotes in vitro se han obtenido usando citocininas solas o combinadas con auxinas.

Se tienen resultados con la interacción de dos citocininas 6-benzylaminopurine (BAP) y 2isopentenil-adenina (2-iP), en combinación con bajos niveles de auxinas; en otros casos con la interacción de citocinina-auxina como: BAP-ácido $\alpha$-naftalenacético (ANA), BAP-ácido indol-3butírico (AIB), 6-furfuryl aminopurine (KIN)-ANA o ácido indol-3-butírico (AIB). Esta variedad de respuestas es función del genotipo y medio de cultivo utilizado. Gonçalves et al. (2016) mencionan que las citocininas (CK) y auxinas (AUX) han sido utilizadas para regular el crecimiento y desarrollo de tejidos vegetales, pero aún no está claro cómo éstos compuestos influyen en la actividad metabólica de las especies in vitro, donde las concentraciones de CK de 0 hasta $20 \mu \mathrm{M}$ pueden influir en la funcionalidad del fotosistema (PS) II, en la anatomía de las hojas, densidad estomatal, producción de brotes a partir de meristemos o yemas axilares.

Considerando que el cultivo in vitro de tejidos vegetales se ha convertido en una herramienta muy útil para acelerar los procesos de producción de plantas, su factibilidad dependerá del manejo de los factores que favorecen este proceso, como el medio de cultivo utilizado donde la concentración de nutrientes, hormonas, horas de luz, temperatura, $\mathrm{pH}$, humedad relativa, suministro de oxígeno, entre otros influyen en el desarrollo de las plantas; por lo que la respuesta morfogenética del explante dependerá de la estandarización del protocolo de micropropagación.

Con el propósito de realizar acciones de conservación ex situ, promover el uso y potenciación de material nativo de interés ornamental (Espinoza-Flores et al., 2003; Granada, 2014; Gámez et al., 2016), como la biznaga de ornato 'cono invertido de Viereck' Turbinicarpus viereckii subsp. major (Glass \& R.A. Foster) Glass, originaria del norte de México, que pertenece a la subfamilia Cactoideae y Tribu Cacteae (Guzmán et al., 2003; Hunt, 2006; Tropicos, 2019) y que se encuentra en estatus de riesgo de protección especial (Pr) de acuerdo con la NOM-059-ECOL-2010 (SEMARNAT, 2010), se hizo necesario evaluar otros esquemas de propagación no convencional para regenerar esta especie mediante el cultivo in vitro, planteando como objetivo de esta investigación el definir el tipo y dosis de citocininas, así como la relación citocinina-auxina que influyen en la etapa de multiplicación, para seleccionar la relación de fitohormonas que promueve el mayor número de brotes in vitro sin perder la estabilidad del explante y estandarizar el protocolo de micropropagación para regenerar esta especie y producir plantas en cantidades suficientes. 


\section{Materiales y métodos}

La investigación se realizó en las instalaciones del Laboratorio de Cultivo de Tejidos Vegetales (LCTV) del Campo Experimental Saltillo CIRNE-INIFAP, ubicado en el municipio de Saltillo, Coahuila, México.

Material vegetal. En la primera etapa se utilizaron vitroplantas germinadas in vitro a partir de semilla colectada en el verano de 2016 en el municipio de Guadalcázar en San Luis Potosí.

Explantes. Cuando las vitroplantas alcanzaron una altura de $10 \mathrm{~mm} \pm 1$ se hicieron dos cortes para eliminar la zona distal y proximal del tallo, utilizando los segmentos intermedios del tallo (SIT) como explantes. Este tipo de explante se usó en todos los subcultivos posteriores (Figura 1).

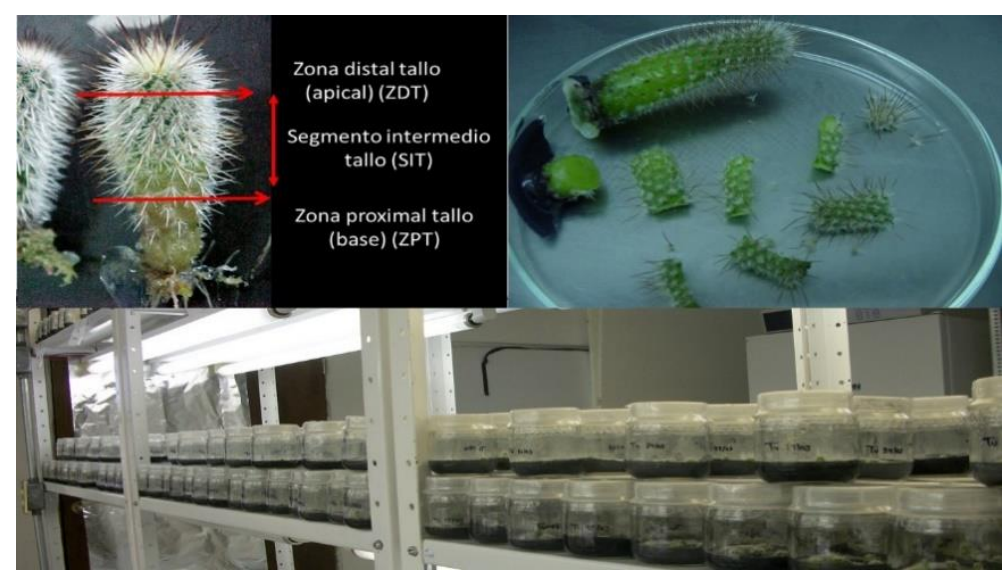

Figura 1. Obtención de explantes y establecimiento de tratamientos en la multiplicación de la biznaga de ornato 'cono invertido de Viereck' (Turbinicarpus viereckii subsp. major (Glass \& R. A. Foster) Glass).

A partir del segundo subcultivo se usaron los SIT obtenidos a partir de la zona distal de tallo (ZDT) (apical) mismos que se subcultivaron y crecieron en el medio Murashige-Skoog (MS; Murashige y Skoog, 1962) como medio base, reduciendo la mitad (50\%) su concentración de macrosales y sin reguladores de crecimiento, para obtener nuevos segmentos intermedios del tallo (SIT) y producir los explantes requeridos en los siguientes subcultivos y continuar con la multiplicación.

Multiplicación de brotes. Los segmentos intermedios del tallo (SIT) usados como explantes fueron establecidos en el medio Murashige-Skoog (MS; Murashige y Skoog, 1962) al 50\% de su concentración de macrosales, suplementado con $100 \mathrm{mg} \mathrm{L}^{-1}$ de mio-inositol (SIGMA ${ }^{\circledR}$ ), $0.4 \mathrm{mg}$ $\mathrm{L}^{-1}$ de tiamina-HCl (SIGMA $\left.{ }^{\circledR}, \mathrm{T}-3906\right), 1 \mathrm{mg} \mathrm{L}^{-1}$ de piridoxina -HCL (SIGMA $\left.{ }^{\circledR}, \mathrm{P}-8666\right), 30 \mathrm{~g}$ $\mathrm{L}^{-1}$ de sacarosa $\left(\right.$ SIGMA $\left.^{\circledR}, \mathrm{K}-0753\right)$ y $7 \mathrm{~g} \mathrm{~L}^{-1}$ de agar $\left(\right.$ SIGMA $\left.^{\circledR}, \mathrm{A}-1296\right)$, con $200 \mathrm{mg} \mathrm{L}^{-1}$ de carbón activado. El pH de medio se ajustó a 5.7 y esterilizó previamente en autoclave marca Felisa a $1.2 \mathrm{~kg} \mathrm{~cm}^{-2}$ de presión a $121{ }^{\circ} \mathrm{C}$ durante $15 \mathrm{~min}$.

Mediante un diseño experimental completamente al azar con arreglo factorial, se evaluaron dos citocininas: BAP y KIN en cuatro concentraciones $2.5,5,7.5$ y $10 \mathrm{mg} \mathrm{L}^{-1}$ en interacción con dos auxinas: ANA y AIB en una relación citocinina-auxina de 10:1. Se establecieron cinco SIT por 
frasco, con 10 repeticiones por tratamiento y evaluaron en total 16 tratamientos. Esta evaluación se repitió cinco veces mediante subcultivos sucesivos de 2017 a 2019 en el LCTV evaluando cada diez semanas el número de brotes por explante $(\mathrm{Nb})$ y la altura del brote $(A b, \mathrm{~mm})$.

Análisis estadístico. Las variables evaluadas se analizaron mediante el procedimiento PROC GLM del sistema de análisis estadístico SAS, (Versión 9.4) (SAS, 2019), realizando un análisis de varianza (Anova) para determinar el efecto de las fitohormonas (citocininas y auxinas), influencia de la concentración y una prueba de rango múltiple Tukey $(p \leq 0.05)$ considerando los tratamientos como efectos independientes para seleccionar el mejor tratamiento.

Condiciones de incubación. El material vegetativo se estableció en el cuarto de incubación del LCTV considerando un fotoperíodo de $14 \mathrm{~h}$ luz por 10 de oscuridad, con una intensidad luminosa de 9-10 $\mu \mathrm{mol} \mathrm{seg}{ }^{-1} \mathrm{~m}^{-2} \mathrm{y}$ una temperatura de $25 \pm 2{ }^{\circ} \mathrm{C}$.

Aclimatación y transferencia a suelo. Los brotes obtenidos se individualizaron y subcultivaron en un medio MS al 50\%, de sus componentes adicionando $1 \mathrm{~g} \mathrm{~L}^{-1}$ de carbón activado para inducir el enraizamiento y luego se pasaron a su aclimatación. Las vitroplantas enraizadas fueron lavadas y tratadas con $1 \mathrm{~g} \mathrm{~L}^{-1}$ de fungicida sistémico comercial con ingrediente activo $\mathrm{N}$-(triclorometiltio) ciclohex-4-eno-1,2-dicarboximida previo al trasplante en macetas de $0.15 \mathrm{~L}$ de capacidad $(6.5 \mathrm{x}$ 5.6 x $5.6 \mathrm{~cm}$ ). Como sustrato se usó una mezcla esterilizada en autoclave (Marca Sumi) a una temperatura de $120{ }^{\circ} \mathrm{C}$ y 1.5 libras de presión compuesta de arena + peat moss+agrolita en relación $3: 2: 1$.

\section{Resultados y discusión}

Estabilidad del cultivo in vitro. Aunque en el Anova existieron diferencias significativas $(\mathrm{F}=60.31$, $p=0.0001)$ entre evaluaciones, los parámetros estadísticos referentes a un alto coeficiente de determinación $\left(\mathrm{R}^{2}\right)$ de 0.72 , menor coeficiente de variación $(\mathrm{CV})$ de 26.4 y diferencia mínima significativa (DMS) de 0.44 mostraron que los segmentos intermedios del tallo (SIT) usados como explante pueden expresar una respuesta morfogenética bajo la influencia de los tratamientos.

En condiciones in vitro, se pueden obtener de 1 hasta 22 brotes por explante, siendo los subcultivos III y IV donde en promedio se obtuvo un $\mathrm{Nb}$ de $9.22 \pm 1$ brotes por explante (Figura 2a). Aunque existieron diferencias entre el primer y último de los cinco subcultivos, los resultados fueron relevantes para un sistema de producción masivo, el cual puede mantenerse por más de tres años y disminuir su producción a partir de la quinta multiplicación clonal, cuestión que debe ser considerada en la programación de la producción a escala comercial.

En altura de brotes $(A b)$ también se encontraron diferencias significativas entre evaluaciones $(\mathrm{F}=$ 427.45, $p=0.0001)$, registrando un coeficiente de determinación $\left(\mathrm{R}^{2}\right)$ de 0.50 , un coeficiente de variación $(\mathrm{CV})$ de 32.65 y una diferencia mínima significativa (DMS) de 0.25 . En condiciones in vitro la $A b$ va de 1 hasta $18 \mathrm{~mm}$, siendo los subcultivos III y IV donde en promedio se obtuvo una $A b$ de $7.56 \pm 1 \mathrm{~mm}$; sin embargo, el tamaño de los brotes obtenidos en todas las evaluaciones realizadas pudo manipularse por el operador al realizar el subcultivo (Figura 2a). 

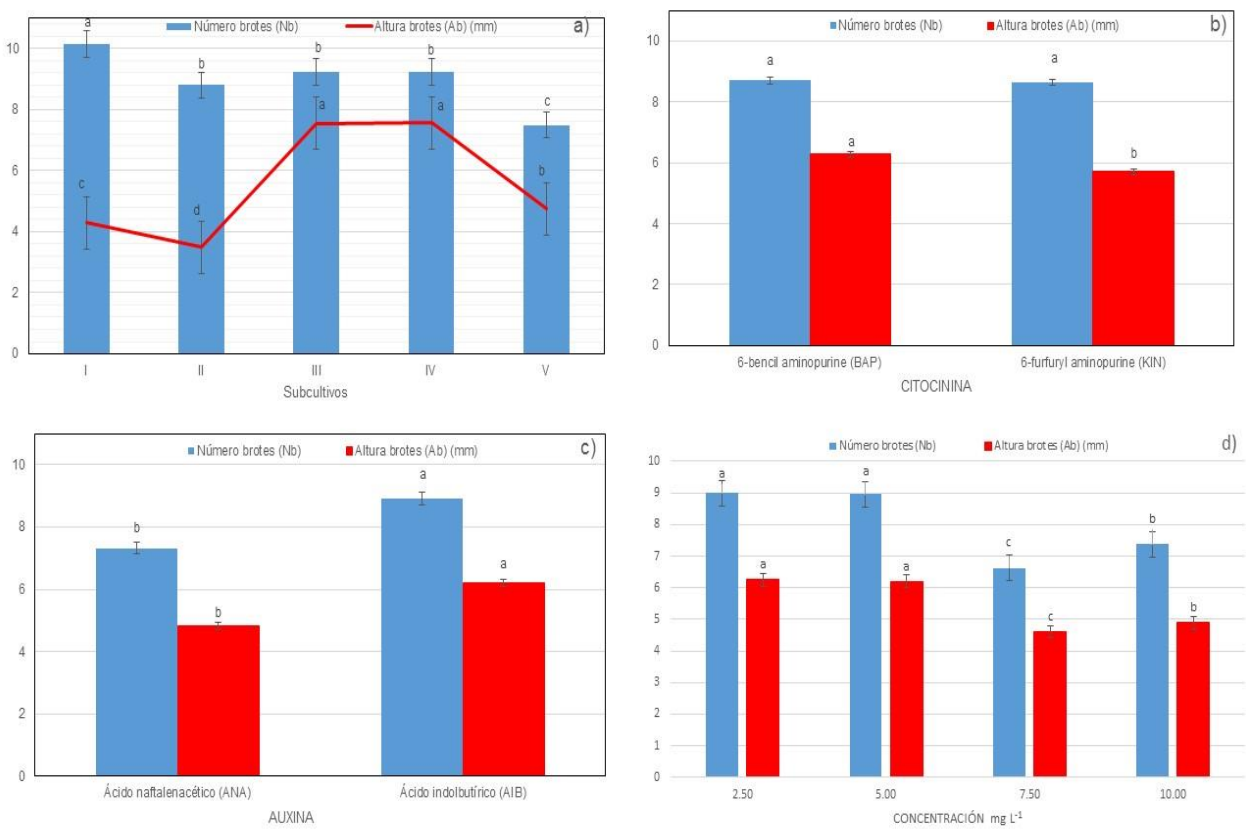

Figura 2. Número de brotes por explante $(N b)$ y altura de brote $(A b)$ en la multiplicación in vitro de la biznaga de ornato 'cono invertido de Viereck' (Turbinicarpus viereckii subsp. major (Glass \& R. A. Foster) Glass). a) influencia de la estabilidad del subcultivo in vitro; b) influencia del tipo de citocininas; c) influencia del tipo de auxinas; y d) influencia de la concentración.

Los resultados registrados en cada una de las evaluaciones realizadas mostraron que los explantes SIT son estables en el cultivo in vitro siendo un tejido vegetal organizado con yemas axilares, a partir del cual se puede regenerar la biznaga 'cono invertido de Viereck' T. viereckii subsp. major.

Influencia del tipo de citocininas. Al analizar el total de los subcultivos el Anova no muestra diferencias significativas $(p \leq 0.05$ ) entre las citocininas evaluadas en $N b$; sin embargo, con el medio de cultivo MS al 50\% con KIN se obtuvo la mayor respuesta, registrando en promedio en $\mathrm{Nb}$ con 8.69 brotes por explante (Figura $2 b$ ).

La tasa de multiplicación para la biznaga $T$. viereckii subsp. major con ambas citocininas supera a la obtenida en $M$. craigii (4.65), $M$. formosa (4.42), M. obscura (4.78) y M. uncinata (5.25) (PérezMolphe-Balch et al., 1998), M. oteroi (5.3) (Castro-Gallo et al., 2002) y es similar con la reportada para biznaga 'cono invertido de Knuth' (Turbinicarpus knuthianus (Boed.) John y Ř́ha (Villavicencio et al., 2011). En $A b$ si se registró una diferencia estadística significativa $(p \leq 0.05)$ entre citocininas, donde la BAP es la fitohormona que influye en esta variable con un $A b$ de 6.27 mm superando en tamaño a los brotes obtenidos con KIN (Figura 2b).

En otras especies de cactáceas como Epithelantha micromeris var. micromeris, Strombocactus disciformis, y T. schmiedickeanus var. klinkerianus (Soltero y Portillo, 2015) y T. knuthianus (Villavicencio et al., 2011) también se ha usado la KIN para el cultivo in vitro, al igual que en otras ornamentales, como orquídeas Laeliocattleya (Orchidaceae) (Gonçalves et al., 2016) y café (Coffe arabiga) (Cantos-Cevallos et al., 2018), mostrando que la respuesta mofogenética depende de la citocinina utilizada y del genotipo. La KIN es de origen natural derivada de purinas o adeninas y 
tiene la capacidad de producir un efecto en la división celular e inducir una respuesta morfogénica en el explante, activando las yemas axilares o meristemos areolares que inducen a la formación de brotes de la biznaga $T$. viereckii subsp. major como también ha ocurrido en Mammillaria schiedeana schiedeana (Soria-Campos et al., 2013).

Influencia del tipo de auxinas. Existieron diferencias significativas $(p \leq 0.05)$ entre las auxinas evaluadas, siendo el ácido indolbutírico (AIB) el que influyó en la respuesta morfogenética tanto en el $\mathrm{Nb}$ como en $\mathrm{Ab}$.

Con esta auxina se obtuvo mayor respuesta en $\mathrm{Nb}$ registrando en promedio 8.92 brotes por explante, con una $A b$ de $6.20 \mathrm{~mm}$ superando el efecto generado con el ácido naftalenacético (ANA), en donde ambas variables registraron menor promedio, con un $N b$ de 7.3 brotes por explante y una $A b$ de 4.5 $\mathrm{mm}$ ) (Figura 2c).

El AIB también se ha utilizado para la inducción de brotes de Epithelantha micromeris var. micromeris, donde se han obtenido hasta 13 brotes por explante (Villavicencio et al., 2012), mientras que el uso del ANA ha tenido efecto en otras cactáceas del género Mammillaria spp. (Pérez-Molphe-Balch et al., 1998; Castro-Gallo et al., 2002; Giusti et al., 2002; Ramírez-Malagón et al., 2007; Soria-Campos et al., 2013) registrando una altura semejante a la reportada en este trabajo.

El mayor número de brotes con menor tamaño es un efecto que ocurre con frecuencia en la micropropagación, como lo refieren Mata-Rosas et al. (2001); Pérez-Molphe-Balch, et al. (2015), por lo que siempre es adecuado evaluar varias combinaciones de citocinina-auxina para determinar el equilibrio entre las variables de respuesta que se buscan en la etapa de multiplicación in vitro de la especie de interés. Esto para asegurar una propagación intensiva, dar continuidad a la etapa de multiplicación y garantizar la supervivencia durante la aclimatización.

Influencia de la concentración. Independientemente del tipo de citocinina utilizada, existieron diferencias altamente significativas entre concentraciones $(p \leq 0.01)$. Las concentraciones de $2.5 \mathrm{y}$ $5 \mathrm{mg} \mathrm{L}^{-1}$ fueron estadísticamente iguales, superando al resto de las concentraciones evaluadas, con un $N b$ de 8.99 brotes/explante y una $A b$ de $6.24 \mathrm{~mm}$ (Figura 2d).

Influencia del tipo de concentración y citocinina. El medio base MS al 50\% adicionado con $2.5 \mathrm{mg}$ $\mathrm{L}^{-1}$ de KIN y $5 \mathrm{mg} \mathrm{L}^{-1}$ de BAP, fueron estadísticamente iguales, superando al resto de las concentraciones evaluadas. Ambas citocininas y concentraciones promovieron la inducción de brotes con un $\mathrm{Nb}$ de 9.19 y 9.09 brotes por explante, respectivamente (Cuadro 1).

La diferencia entre ambas concentraciones y tipo de citocininas radicó en la altura de brotes, donde la mayor respuesta se obtuvo con $5 \mathrm{mg} \mathrm{L}^{-1}$ de BAP registrando una $A b$ de $6.55 \mathrm{~mm}$. Estos resultados mostraron que los SIT fueron más compatibles con KIN, una fitohormona natural, donde a menor concentración en el tejido vegetal se generó mayor respuesta en la inducción de brotes, a diferencia de los SIT que fueron expuestos con BAP, que es una citocinina sintética donde la mayor inducción de brotes se obtuvo al duplicar la concentración (Cuadro 1). 
Cuadro 1. Tipo de citocinina y concentración en la multiplicación in vitro de la biznaga de ornato 'cono invertido de Viereck' (Turbinicarpus viereckii subsp. major (Glass \& R. A. Foster) Glass).

\begin{tabular}{|c|c|c|c|c|c|}
\hline \multirow{2}{*}{ Citocinina } & \multirow{2}{*}{$\left(\mathrm{mg} \mathrm{L}^{-1}\right)$} & \multicolumn{2}{|c|}{ Número de brotes $(\mathrm{Nb})$} & \multicolumn{2}{|c|}{ Altura de brotes $(A b)$} \\
\hline & & Media & Desviación estándar & Media & Desviación estándar \\
\hline \multirow[t]{4}{*}{ 6-bencil aminopurine (BAP) } & 2.5 & 8.78 & \pm 3.25 & 6.7 & \pm 3.02 \\
\hline & 5 & 9.09 & \pm 4.21 & 6.55 & \pm 2.8 \\
\hline & 7.5 & 7.24 & \pm 3.65 & 4.72 & \pm 1.09 \\
\hline & 10 & 8.03 & \pm 4.46 & 4.86 & \pm 1.86 \\
\hline \multirow{4}{*}{$\begin{array}{l}\text { 6-furfuryl aminopurine } \\
\text { (KIN) }\end{array}$} & 2.5 & 9.19 & \pm 3.58 & 5.85 & \pm 2.35 \\
\hline & 5 & 8.83 & \pm 3.33 & 5.85 & \pm 2.68 \\
\hline & 7.5 & 5.62 & \pm 2.34 & 4.44 & \pm 1.18 \\
\hline & 10 & 6.45 & \pm 2.57 & 4.92 & \pm 1.29 \\
\hline
\end{tabular}

El efecto del tipo de citocinina y concentración en el número y altura de brotes también se ha reportado en biznagas de tipo globoso, en donde se han aplicado de 3 hasta $10 \mathrm{mg} \mathrm{L}^{-1}$ de KIN (Soltero y Portillo, 2015) y de 0.5 hasta $2 \mathrm{mg} \mathrm{L}^{-1}$ cuando se ha utilizado BAP (Soria-Campos et al., 2013).

Los resultados muestran que el control hormonal influye en la diferenciación del explante, como lo refieren Pérez-Molphe-Balch y Dávila-Figueroa (2002); Dávila et al. (2005); Ascough y Van Staden (2010); De la Rosa-Carrillo et al. (2012). Este control tuvo un efecto positivo en el cultivo in vitro logrando que la conservación ex situ sea factible tanto para la cactácea en estudio $T$. viereckii subsp. major, como para otras especies, como el viejito Cephalocereus senilis (Haw.) Pfeiff. (Choreño et al., 2002), pitaya Stenocereus stellatus (Pfeiff.) Riccob. (Martínez-Villegas et al., 2011), mamilaria Mammillaria voburnensis Scheer (Ordoñez, 2003), cactus alcachofa Obregonia denegrii Frič \& A. Berger (Cardarelli et al., 2010) y minicactus Turbinicarpus spp.

Influencia de los tratamientos. Al realizar la prueba de medias de Tukey $(p \leq 0.05)$ a los tratamientos como efectos independientes, tomando en consideración la interacción entre el tipo de citocinina y auxina con sus diferentes concentraciones evaluadas, se determinó que existen dos tratamientos que pueden ser utilizados en la etapa de multiplicación. El medio MS al 50\% adicionado con $5 \mathrm{mg}$ $\mathrm{L}^{-1}$ de $\mathrm{BAP}+0.5 \mathrm{mg} \mathrm{L}^{-1}$ de AIB y el adicionado con $2.5 \mathrm{mg} \mathrm{L}^{-1}$ de $\mathrm{KIN}+0.25 \mathrm{mg} \mathrm{L}^{-1}$ de AIB fueron estadísticamente igual superando en $\mathrm{Nb}$ al resto de los tratamientos evaluados, registrando un $\mathrm{Nb}$ de hasta 9.25 brotes por explante; sin embargo, con el segundo tratamiento se obtienen brotes con mayor tamaño, con una $A b$ de $6.73 \mathrm{~mm}$, lo que facilita una mejor manipulación de estos al realizar el subcultivo (Cuadro 2).

Estos resultados muestran que la interacción citocinina:auxina en relación 10:1 influye en la morfogénesis del explante de esta especie, regulando la división y crecimiento celular de las yemas axilares para la inducción de brotes (Figura 3). 
Cuadro 2. Relación citocina-auxina en la activación de yemas axilares en la etapa de multiplicación in vitro de la biznaga de ornato 'cono invertido de Viereck' (Turbinicarpus viereckii subsp. major (Glass \& R. A. Foster) Glass).

\begin{tabular}{cccc}
\hline & Tratamiento & Núm. de brotes $(N b)$ & Altura de brotes $(A b)(\mathrm{mm})$ \\
\hline 1 & $2.5 \mathrm{mg} \mathrm{L}^{-1} \mathrm{BAP}+0.25 \mathrm{mg} \mathrm{L}^{-1} \mathrm{ANA}$ & $7.57 \mathrm{~d}$ & $5.04 \mathrm{~d}$ \\
2 & $5 \mathrm{mg} \mathrm{L}{ }^{-1} \mathrm{BAP}+0.5 \mathrm{mg} \mathrm{L}^{-1} \mathrm{ANA}$ & $7.4 \mathrm{~d}$ & $4.63 \mathrm{ef}$ \\
3 & $7.5 \mathrm{mg} \mathrm{L}^{-1} \mathrm{BAP}+0.75 \mathrm{mg} \mathrm{L}^{-1} \mathrm{ANA}$ & $5.74 \mathrm{f}$ & $4.95 \mathrm{e}$ \\
4 & $10 \mathrm{mg} \mathrm{L}^{-1} \mathrm{BAP}+1 \mathrm{mg} \mathrm{L}^{-1} \mathrm{ANA}$ & $7.57 \mathrm{~d}$ & $5.3 \mathrm{~cd}$ \\
5 & $2.5 \mathrm{mg} \mathrm{L}^{-1} \mathrm{BAP}+0.25 \mathrm{mg} \mathrm{L}^{-1} \mathrm{AIB}$ & $8.85 \mathrm{~b}$ & $6.8 \mathrm{a}$ \\
6 & $5 \mathrm{mg} \mathrm{L}^{-1} \mathrm{BAP}+0.5 \mathrm{mg} \mathrm{L}^{-1} \mathrm{AIB}$ & $9.24 \mathrm{a}$ & $6.07 \mathrm{~b}$ \\
7 & $7.5 \mathrm{mg} \mathrm{L}^{-1} \mathrm{BAP}+0.75 \mathrm{mg} \mathrm{L}^{-1} \mathrm{AIB}$ & $8.3 \mathrm{c}$ & $4.55 \mathrm{f}$ \\
8 & $10 \mathrm{mg} \mathrm{L}^{-1} \mathrm{BAP}+1 \mathrm{mg} \mathrm{L}^{-1} \mathrm{AIB}$ & $8.36 \mathrm{c}$ & $4.54 \mathrm{f}$ \\
9 & $2.5 \mathrm{mg} \mathrm{L}^{-1} \mathrm{KIN}+0.25 \mathrm{mg} \mathrm{L}^{-1} \mathrm{ANA}$ & $8.87 \mathrm{ab}$ & $4.54 \mathrm{f}$ \\
10 & $5 \mathrm{mg} \mathrm{L}^{-1} \mathrm{KIN}+0.5 \mathrm{mg} \mathrm{L}^{-1} \mathrm{ANA}$ & $7.54 \mathrm{~d}$ & $5.07 \mathrm{~d}$ \\
11 & $7.5 \mathrm{mg} \mathrm{L}^{-1} \mathrm{KIN}+0.75 \mathrm{mg} \mathrm{L}^{-1} \mathrm{ANA}$ & $5.89 \mathrm{f}$ & $4.06 \mathrm{~g}$ \\
12 & $10 \mathrm{mg} \mathrm{L}^{-1} \mathrm{KIN}+1 \mathrm{mg} \mathrm{L}^{-1} \mathrm{ANA}$ & $6.5 \mathrm{e}$ & $5.24 \mathrm{~d}$ \\
13 & $2.5 \mathrm{mg} \mathrm{L}^{-1} \mathrm{KIN}+0.25 \mathrm{mg} \mathrm{L}^{-1} \mathrm{AIB}$ & $9.25 \mathrm{a}$ & $6.73 \mathrm{a}$ \\
14 & $5 \mathrm{mg} \mathrm{L}^{-1} \mathrm{KIN}+0.5 \mathrm{mg} \mathrm{L}^{-1} \mathrm{AIB}$ & $9 \mathrm{ab}$ & $5.95 \mathrm{c}$ \\
15 & $7.5 \mathrm{mg} \mathrm{L}^{-1} \mathrm{KIN}+0.75 \mathrm{mg} \mathrm{L}^{-1} \mathrm{AIB}$ & $5.21 \mathrm{~g}$ & $5.01 \mathrm{~d}$ \\
16 & $10 \mathrm{mg} \mathrm{L}^{-1} \mathrm{KIN}+1 \mathrm{mg} \mathrm{L}^{-1} \mathrm{AIB}$ & $6.42 \mathrm{e}$ & $4.71 \mathrm{ef}$ \\
& $\mathrm{r}$ & 0.76 & 0.69 \\
& $\mathrm{CV}$ & 18.36 & 26.1 \\
& $\mathrm{STD}$ & 2.85 & 2.9 \\
& $\mathrm{x}$ & 7.61 & 5.07
\end{tabular}

Medias con la misma letra en cada columna son iguales estadísticamente (Tukey $p \leq 0.05$ ).

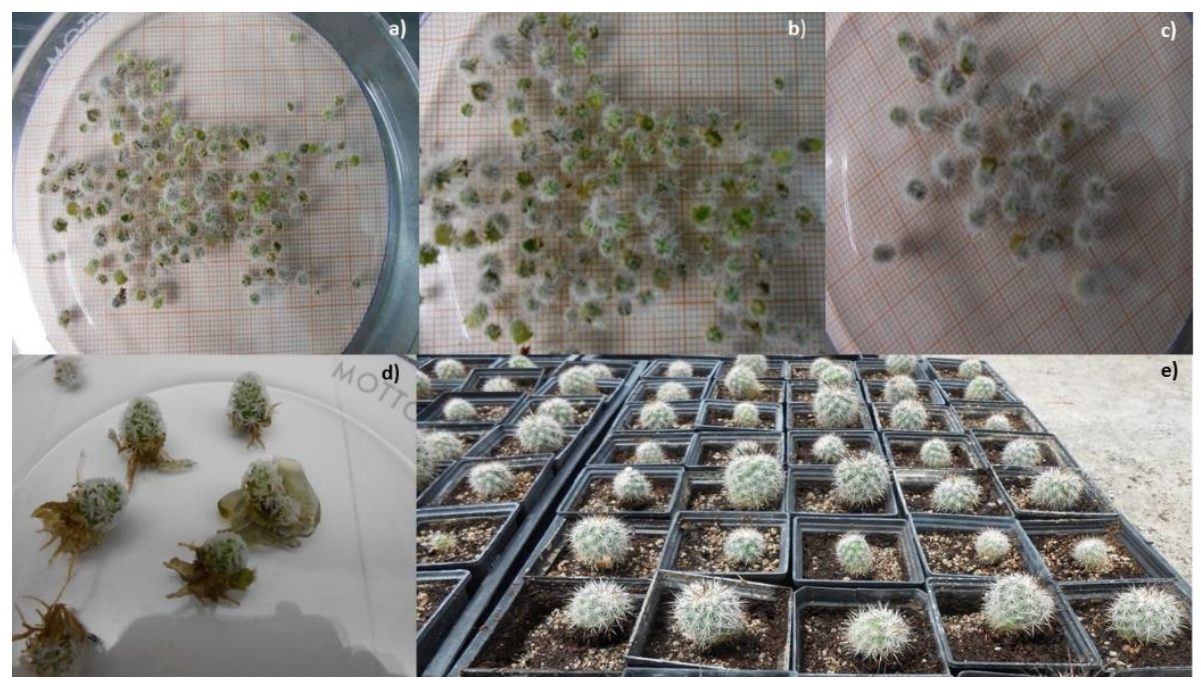

Figura 3. Multiplicación de la biznaga de ornato 'cono invertido de Viereck' (Turbinicarpus viereckii subsp. major (Glass \& R. A. Foster) Glass). a, b y c) inducción de brotes; d) vitroplantas; y e) plantas aclimatadas. 
La interacción KIN:AIB es similar a la reportada por Finti et al. (2012); Pacheco (2015); Velázquez y Soltero (2001); Villavicencio et al. (2020), quienes encontraron que la fuente de citocinina tiene efectos significativos sobre la producción de brotes en la biznaga 'nido de golondrina' Epithelantha micromeris var. micromeris (Engelm.) F. A. C. Weber ex Britton \& Rose, nopal Opuntia ficusindica (L.) Mill. y 'sacasil' Echinocereus poselgeri Lem. por lo que seconsidera este aspecto en la micropropagación de cactáceas, dado que la eficiencia del tratamiento para la inducción de brotes, como el coeficiente de brotación, son importantes en la etapa de multiplicación in vitro para alcanzar los resultados finales en la micropropagación, de esta etapa dependerá la cantidad de explantes que se establezcan, número de brotes que se generen y vitroplantas que se produzcan.

Al aumentar la concentración en la interacción BAP:AIB y KIN:AIB la tasa de multiplicación disminuye. La menor respuesta morfogenética en las diferentes concentraciones evaluadas se obtuvo con las interacciones BAP:ANA y KIN:ANA.

Esta respuesta indica que el ácido naftalenacético (ANA) es una auxina que estimuló escasamente el crecimiento en las yemas axilares de los explantes de T. viereckii subsp. major. En otras especies, como Mimosa pudica (Bianchetti et al., 2017) la asociación BAP:ANA también registró una baja inducción de brotes, como el efecto reportado en la presente investigación.

En cactáceas como Turbinicarpus pseudomacrochele y Strombocactus disciformis, Soltero y Portillo (2015) refieren que la interacción KIN:ANA puede presentar un efecto significativo cuando se aplica en concentración de 3 a $10 \mathrm{mg} \mathrm{L}^{-1}$ de $\mathrm{KIN}$, combinada con una baja concentración $0.02 \mathrm{mg} \mathrm{L}^{-1}$ de ANA, por lo que es necesario considerar dichos factores en este proceso, ya que la respuesta morfogenética del explante dependerá significativamente de ésta interacción y del resto de los componentes del medio de cultivo.

\section{Conclusiones}

La regeneración de esta especie en estatus de riesgo, así como su conservación ex situ puede realizarse con la multiplicación in vitro, donde la relación citocinina-auxina influye en la capacidad morfogenética y en la estabilidad del explante, a partir del cual se puede inducir la proliferación de brotes axilares.

\section{Agradecimientos}

A la convocatoria de proyectos fiscales de INIFAP por el apoyo al proyecto con registro SIGI: 11375835151: titulado: 'Estrategias de innovación tecnológica para la conservación ex situ de cactáceas ornamentales del desierto Chihuahuense un recurso fitogenético nativo de México’.

\section{Literatura citada}

Aggarwal, D.; Upadhyay, S. K.; Kumar, K.; Sehrawat, N.; Tuli, H. S. and Singh, R. 2018. Effects of plant growth regulators on in vitro propagation of economically important ornamental plant Rosa hybrida L. Asian J. Biol Life Sci. 9(2):227-233. Doi :10.5530/ajbls.2018.9.35.

Ascough, G. D. and Staden, V. J. 2010. Micropropagation of Albuca bracteata and A. nelsonii indigenous ornamentals with medicinal value. South Afr. J. Bot. 76(3):579-584. https://www.researchgate.net/publication/337387811. 
Bianchetti, R. E.; Ferrara, R. C.; Sacramento, P. V.; Floriano, D. F.; Mystica, S. de O A.; Condé, E. F J. and Pereira, P. P. H. 2017. An improved protocol for in vitro propagation of the medicinal plant Mimosa pudica L. Afr. J. Biotechnol. 16(9):418-428. Doi:10.5897/ AJB2016.15831.

Cantos-Cevallos, G.; Pinargote-Choez, J. y Palma-Ponce R. 2018. Influencia de la fitohormona kinetina en el crecimiento de plántulas de Coffea arabiga L. injertadas sobre patrón robusta en vivero. Rev. Cubana Ciencias Forestales. Pinar del Río. 6(2):134-145. http://scielo.sld. cu/scielo.php?script=sci-arttext\&pid=s231034692018000200134\&lng=es\&nrm=iso.

Cardarelli, M.; Borgognone, D. y Colla, G. 2010. Propagación in vitro de Obregonia denegrii (Cactaceae). Propagación de plantas ornamentales. 10(1):29-36. https://www.researchgate. net/publication/262261883.

Castro-Gallo, I.; Meza-Rangel, E.; Pérez-Reyes, M. E. y Pérez-Molphe, B. M. E. 2002. Propagación in vitro de 10 especies mexicanas de cactáceas. Sciantiae Naturae. 4(2):5-24.

Choreño, T. J.; González, M. R. H.; Terrazas, T. S. y Hernández, L. A. 2002. Propagación in vitro de Cephalocereus senilis Haworth Pfeiffer a partir de areolas. Rev. Chapingo. Ser. Hortic. 8(2):183-196. https://doi.org/10.5154/r.rchsh.2001.01.008.

Dávila, F. C. A.; De La Rosa-Carrillo, M. L. and Pérez, B. M. 2005. In vitro propagation of eight species or subspecies of Turbinicarpus (Cactaceae). In vitro Cel. Dev. Biology-Plant. 41(4):540-545. https://doi.org/10.1079/IVP2005668.

De la Rosa-Carrillo, M. L.; Domínguez-Rosales, M. S.; Pérez-Reyes, M. E. y Pérez-Molphe-Balch, E. 2012. Cultivo y propagación in vitro de cactáceas amenazadas del género Turbinicarpus. Interciencia. 37(2):114-120. http://www.redalyc.org/articulo.oa?id=33922717006.

Espinoza-Flores, A.; González-Rosas, H. y Mejía-Muñoz, J. 2003. La comercialización de plantas en peligro de extinción. In: plantas nativas de México con potencial ornamental. Universidad Autónoma, Chapingo (UACH). AMEHOAC. Asociación Mexicana de Horticultura Ornamental, AC. Texcoco, Estado de México, México. 199-217 pp.

Finti, I. A.; Boullani, E. R.; Ayadi, E. F.; Aabd, A. N. and Mousadik, E. A. 2012. Micropropagation in vitro of Opuntia Ficus-indica in south of Morocco. Int. J. Chem. Biochem. Sci. 1:6-10. http://www.iscientific.org/wp-content/uploads/2018/02/2-IJCBS-12-1-06.pdf.

Gámez, M.; Villavicencio, O. E. E. G.; Serrato, M. A. C.; Mejía, J. M. M.; Treviño, G. C.; Martínez, L. G.; Rodríguez, M. O.; Granada, L. C.; Flores, M. C.; Reyes, J. S.; Islas, M. L.; Salome, E. C.; Menchaca, R. A. G.; Espadas, C. M. M.; Hernández, L. S.; Vázquez, L.M. C.; Martínez, G. F. M.; Vargas, O. P. y Ríos, E. S. 2016. Conservación y aprovechamiento sostenible de especies ornamentales nativas de México. Servicio Nacional de Inspección y Certificación de Semillas (SNICS)-Universidad Autónoma Chapingo (UACH). Texcoco, Estado de México, México. 152 p. https://www.gob.mx/cms/uploads/attachment/ file/172778/Conservaci_n_y_aprovechamiento_sostenible_de_especies_ornamentales_de_M_ xico.pdf.

Giusti, P.; Vitti, D.; Fiocchetti, F.; Colla, G.; Saccardo, F. and Tucci, M. 2002. In vitro propagation of three endangered cactus species. Sci. Hortic. 95(4):319-332. https://www.sciencedirect.com/science/article/abs/pii/S0304423802000316?via\%3Dihub.

Gonçalves, M. L.; Machado, P. S. M. de Fátima.; Ballesta, P.; Mora, F.; Milaneze, G. M. A. y Aparecida, M. C. 2016. Suplementos orgánicos para el cultivo in vitro del híbrido Laeliocattleya (Orchidaceae). Idesia. 1(34):47-54. http://dx.doi.org/10.4067/S071834292016000100006. 
Granada, C. L. 2014. La importancia del sector ornamental como un potencial de alto contenido de participación social. In: primer simposio nacional plantas ornamentales nativas mexicanas con potencial comercial. SNICS-SINAREFI. Tezoyuca. Morelos México. 10 p.

Guzmán, U.; Arias, M. S. y Dávila, P. P. 2003. Catálogo de cactáceas mexicanas. Universidad Nacional Autónoma de México (UNAM)- Comisión Nacional para el Conocimiento y Uso de la Biodiversidad (CONABIO). México, DF. México. 315 p. https://www.worldcat.org/ title/catalogo-de-cactaceas-mexicanas/oclc/948238461.

Hunt, D. 2006. The new cactus lexicon. descriptions \& illustrations of the cactus family. Compiles and edited by members or the International Cactaceae Systematic Group. England. (Ed.). 373 p. https://www.nhbs.com/the-new-cactus-lexicon-volumes-i-and-ii-book.

Martínez-Villegas, Y. M.; Andrade-Rodríguez, M.; Villegas-Monter, A.; Alia-Tejacal, I.; VillegasTorres O. G. y López-Martínez, V. 2011. Cultivo in vitro de pitayo (Stenocereus stellatus [Pfeiffer] Riccobono). Rev. Chapingo Hortic. 17(3):95-105. https://doi.org/10.5154/r. rchsh. 2011.17.021.

Mata-Rosas, M.; Monroy-De la Rosa, M. A.; Moebius-Goldammer, K. and Chávez-Ávila, V. M. 2001. Micropropagation of Turbinicarpus laui Glass et Foster, an endemic and endangered species. In vitro Cell. Dev. Pl. 37:400-404. https://doi.org/10.1007/s11627-001-0070-6.

Murashige, T. and Skoog, F. 1962. A revised medium for rapid growth and bioassay with tobacco tissue culture. Physiologia Plantarum. 15(3):473-477. http://dx.doi.org/10.1111/j.13993054.1962.tb08052.x.

Ordoñez, M. M. A. 2003. Propagación in vitro de Mammillaria voburnensis Scheer. (Cactaceae) Universidad de San Carlos de Guatemala. Facultad de Ciencias Químicas y Farmacia. Guatemala. 70 p. http://biblioteca.usac.edu.gt/tesis/06/06_2162.pdf.

Pérez-Molphe-Balch, E.; Pérez-Reyes, M. E.; Villalobos-Amador, E.; Meza-Rangel, E.; MoronesRuiz, L. R. and Lizalde-Viramontes, H. J. 1998. Propagation of 21 species of mexican cacti by axillary proliferation. In vitro Cell. Dev. Pl. 34(2):131-135. https://doi.org/10.1007/BF02822777.

Pérez-Molphe-Balch E. and Dávila-Figueroa, C. A. 2002. In vitro propagación de Pelecyphora aselliformis Ehrenberg y P. strobiliformis Werdermann (Cactaceae). In Vitro Cel. Develop. Biol. Plant. 38(1):73-78. https://link.springer.com/article/10.1079\%2FIVP2001248.

Pérez-Molphe-Balch, E.; Santos-Díaz, M. S.; Ramírez-Malagón, R. y Ochoa-Alejo, N. 2015. Cultivo de tejidos de cactus ornamentales. Sci. Agric. 72(6):540-561. https://www.scielo.br/j/sa/a/VtcbrDrxhm8L3yLFDcfV46j/?lang=en.

Ramírez-Malagón, R.; Aguilar-Ramírez, I.; Borodanenko, A.; Pérez-Moreno, L.; Barrera-Guerra, J. L.; Nulez-Palenius, H. G. and Ochoa-Alejo, N. 2007. In vitro propagation of ten thratened species of Mammillaria (Cactaceae). In vitro Cell. Dev. Pl. 43(6):660-665. https://agris.fao.org/agris-search/search.do?recordID=US201500203765.

SEMARNAT. 2010. Secretaría del Medio Ambiente y Recursos Naturales. Norma Oficial Mexicana NOM-059-ECOL-2010. Protección ambiental-especies nativas de México de flora y fauna silvestres-Categorías de riesgo y especificaciones para su inclusión, exclusión o cambio. Lista de especies en riesgo. Diario Oficial de la Federación. 30 de diciembre de 2010. México, DF. 130 p. https://dof.gob.mx.

Soltero, Q. R. y Portillo L. 2015. Micropropagación de cactáceas mexicanas amenazadas. Bol. Nakari. 26(2):13-17. https://www.researchgate.net/publication/285204536. 
Soria-Campos, D.; López-Escamilla, A. L. y Olguín-Santos, L. P. 2013. Propagación in vitro de Mammillaria schiedeana schiedeana (Cactaceae), subespecie endémica y amenazada de extinción de la Barranca de Metztitlán, Hidalgo. Estudios científicos en el estado de Hidalgo y zonas aledañas. Lincoln, NE. Zea Books. 2(16):121-128. https://digitalcommons.unl.edu/cgi/viewcontent.cgi?article=1015\&context=hidalgo.

SAS. 2019. Statistical Analysis System Institute Inc. SAS/STAT ${ }^{\circledR} 14.1$ User's guide second edition. SAS Institute Inc. Raleigh, NC, USA. 238 p.

Tropicos. 2019. Trópicos: Turbinicarpus viereckii subsp. major (Glass \& R.A. Foster) Glass). http://www.tropicos.org/Name/33700793.

Velázquez E. L. E. y Soltero, Q. R. 2001. Micropropagación de Ephithelantha micromeris Engelm. Weber ex Britton et Rose. 46(3):56-62.

Villanueva, F.; Ávila, M.; Mansilla, A.; Abades, S. y Cáceres, J. 2013. Efecto de auxinas y citoquininas en el cultivo de tejido de Ahnfeltia plicata (Hudson) Fries, 1836 (Ahnfeltiales, rhodophyta) de la región de Magallanes. Anales Instituto Patagonia. Punta Arenas, Chile. 1(41):99-111. http://dx.doi.org/10.4067/S0718-686X2013000100009.

Villavicencio-Gutiérrez, E. E.; González-Cortes, A.; Arredondo-Gómez, A.; Iracheta-Donjuan, L.; Comparan-Sánchez, S. y Casique-Valdés, R. 2011. Micropropagación de Turbinicarpus knuthianus (Boed.) John \& Riha cactácea ornamental, amenazada de extinción del Desierto Chihuahuense. Rev. Mex. Cien. Forest. 6(2):37-56. https://doi.org/10.29298/rmcf. v2i6.573.

Villavicencio, G. E. E.; González, C. A. y Carranza, P. M. A. 2012. Micropropagación de Epithelantha micromeris (Engelm.) F.A.C. Weber ex Britt. \& Rose cactácea ornamental y recurso fitogenético del Desierto Chihuahuense. Rev. Mex. Cien. Forest. 14(3):83-99. Doi: https://doi.org/10.29298/rmcf.v3i14.476.

Villavicencio, G. E. E.; Arellano, O. G. y Belmontes, D. V. A. 2020. Micropropagación del órgano pequeño sacasil (Echinocereus poselgeri Lem.) (Fam.: Cactaceae). Acta Hortic. 1288(1):123-130. Doi: 10.17660/ActaHortic.2020.1288.19. 